\title{
Relationships between Affect and Short-Term Life Satisfaction: Considering the Activation Dimension and Balance of Affect
}

\author{
Katsuyuki Yamasaki ${ }^{1} \&$ Kanako Uchida ${ }^{1}$ \\ ${ }^{1}$ Department of Human Development, Naruto University of Education, Naruto, Tokushima, Japan \\ Correspondence: Katsuyuki Yamasaki, Department of Human Development, Naruto University of Education, Naruto, \\ Tokushima, 772-8502, Japan.
}

Received: April 26, 2016

Accepted: May 20, 2016

Available online: May 24, 2016

doi:10.11114/ijsss.v4i7.1629

URL: http://dx.doi.org/10.11114/ijsss.v4i7.1629

\begin{abstract}
The purpose of this study was to examine the relationship between affect and short-term life satisfaction (LS) taking into account the dimensions of activation and balance of PA and NA. The study included 398 students (184 men and 214 women) who completed six questionnaires, three of which were used for another study. The Positive and Negative Affect Schedule was utilized for assessing activated PA and NA. Two subscales (deactivated pleasure and boredom) of the Multiple Mood Scale were used for measuring deactivated PA and NA, and the Short-term Life Satisfaction Scale was used for gauging short-term LS. Correlation analyses and hierarchical multiple regression analyses were conducted to determine the relationships between affect balance (PA-NA and PA/NA) and LS. The results of correlation and scattergram analyses showed that LS displayed positive linear associations with affect balance in both activated and deactivated dimensions. Additionally, hierarchical multiple regression analyses revealed a significant interaction between activated PA and NA in women, and indicated that the positive relationship between activated PA and LS in women was strengthened when levels of activated NA were lower. We discuss the importance of considering subtypes within and relationships between PA and NA and overall implications for health/adjustment.
\end{abstract}

Keywords: positive affect, negative affect, activation dimension of affect, short-term life satisfaction

\section{Introduction}

In recent years, relationships between affect (or emotions) and health (or adjustment) have been topics of intense research in the domains of health psychology and behavioral medicine. Although the detrimental influences of negative affect (NA) on health are well known, an increasing number of studies have revealed that positive affect (PA) plays a crucial and beneficial role in health (see Pressman \& Cohen, 2005, for a review). These phenomena relating affect and health have recently been scrutinized by examining the relations between PA and NA.

For example, the overall affective balance between PA and NA has been shown to be important to health. Since PA and NA are not contrary concepts and display null or low correlations (e.g., Pettit, Kline, Gencoz, Gencoz, \& Joiner, 2001; Watson, Clark, \& Tellegen, 1988), their relative contributions to health deserve examination. Previously, significant interactions between PA and NA were found to influence many health-related outcomes, such as well-being (Cheng, 2006), job performance (Van Yperen, 2003), and breast cancer concerns (Han, Shaw, Hawkins, Pingree, McTavish, \& Gustafson, 2008). Cheng (2006) showed that the enhancing effect of PA on well-being appraisal became stronger when NA is higher. Han et al. (2008) found a tempering effect between writing positive emotion words and breast cancer concerns among those who wrote a higher percentage of negative emotion words. Similarly, Van Yperen (2003) found that the enhancing effect of PA on job performance was significant only when NA was high.

In addition, the effects of affect balance have been investigated in cross-cultural research. For example, Schimmack, Radhakrishnan, Oishi, Dzokoto, and Ahadi (2002) found that affect balance (as represented by the difference score of PA minus NA) was a stronger predictor of life satisfaction in individualistic cultures versus collectivistic ones. Suh, Diener, Oishi, and Triandis (1998) showed that correlations of affect balance with life satisfaction varied across nations, although the relationship was significantly positive in all cases. Thus, it is likely that PA and NA have not only main effects on health and adjustment but interactive effects as well.

Investigations into the effects of affect balance on health also need to consider another dimension of affect termed "activation." According to the two-factor model of affect, the first unrotated factor is interpreted as a Valence dimension 
and the second unrotated factor as an Activation or Arousal dimension (Larsen \& Diener, 1992; Russell, 1980). Thus, PA and NA, which represent the opposite ends of the Valence dimension, also change in the Activation dimension, which allows us to consider different types of PA and NA, i.e., activated PA (e.g., enthusiastic, happy), deactivated PA (e.g., calm, satisfied), activated NA (e.g., nervous, angry), and deactivated NA (e.g., sluggish, sad). It should be noted that the PANAS (Positive and Negative Affect Schedule; Watson et al., 1988), one of the most frequently used questionnaires, measures only activated PA and NA.

In recent years, a few studies have begun to discriminate the two types of affect along the activation axis (e.g., De Dreu, Baas, \& Nijstad, 2008; Nezlek \& Kuppens, 2008; Tsai, Knutson, \& Fung, 2006), and these reports suggest distinct roles for activated and deactivated affect. For example, Nezlek and Kuppens (2008) showed that the suppression of negative emotions was negatively associated with deactivated but not activated NA. De Dreu et al. (2008) revealed that activating moods (i.e., activated PA and NA) lead to more creative fluency and originality compared to deactivating moods (i.e., deactivated PA and NA). In research on cultural variations in affect valuation, Tsai, Knutson, and Fung (2006) found that European American and Asian American individuals valued high-arousal PA more so than Hong Kong Chinese, while Hong Kong Chinese and Asian American individuals value low-arousal PA more so than European Americans. These findings would not have been discovered if measures of PA and NA had not been divided and recorded along the activation axis.

With respect to health and adjustment, differential effects of activated and deactivated affect are plausible. Likewise, the effects of affect balance on health and adjustment could be different between activated and deactivated affect dimensions. However, to the authors' knowledge, no prior research has focused on these differentiations, which prevents us from building hypotheses on them.

Thus, the aim of the present study was to examine the effects of affect balance on health and adjustment. In doing so, we considered both activated and deactivated PA and NA. Moreover, we focused on short-term life satisfaction as a health/adjustment outcome. Investigations into health problems in non-clinical samples require measures sensitive to preconditions that could lead to future disease. One of those candidate measures is life satisfaction, which is a conscious cognitive judgment of one's past whole life in which the criteria for judgment are up to the individual (Pavot \& Diener, 1993). A number of studies revealed that affect balance was positively associated with life satisfaction (Koydemir, Şimşek, Schütz, \& Tipandjan, 2013; Sanjuán, 2011; Zhu, 2015). However, no such previous research considered the activation dimension of affect. Moreover, in the current research, we assessed life satisfaction for the past week, which would represent a more sensitive measure of short-term life satisfaction, since "life" in life satisfaction does not need to be limited to the past whole life.

\section{Method}

\subsection{Participants and Procedure}

Participants were 478 undergraduate students from three universities in Japan. Incomplete data were obtained from 70 participants, while the ages of 10 participants were over seven SDs from the mean age. These data were therefore excluded, so that the final sample included data collected from 398 participants (184 men and 214 women). The mean ages and SDs for these samples were 19.15 $\pm 1.47 \mathrm{yrs}$ for men and $19.84 \pm 1.33 \mathrm{yrs}$ for women.

Participants answered six questionnaires in their classes of various sizes (approximate class sizes varied from 19 to 179 students). Since three of the six questionnaires were used for another study that did not relate to the purposes of the current study, these results are not reported in this paper.

\subsection{Measures}

Activated and Deactivated PA and NA. Activated PA and NA were measured using the Japanese version of the Positive and Negative Affect Schedule (PANAS) (Sato \& Yasuda, 2001). The alphas were .82 and .84 for the PA and NA scales in this study, respectively. The construct validity of these scales was previously established by a study in which PA, NA and neutral affect were experimentally manipulated (Sato \& Yasuda, 2001). Unlike the original version (Watson et al., 1988), the Japanese version contains eight items each for PA (e.g., enthusiastic, proud, and excited) and NA (e.g., afraid, jittery, and scared), which are rated on a 6-point Likert scale (from "not at all" to "extremely"). Thus, scores on the PA and NA scales each ranged from 8 to 48. In this study, participants were instructed to indicate the extent to which each item represented the way they felt over the previous one-week period. Deactivated PA and NA were measured using the subscales of deactivated pleasure and boredom (respectively) from the Multiple Mood Scale (MMS: Terasaki, Kishimoto, \& Koga, 1992). Each subscale contained five items (e.g., relaxed, calm, and placid for PA; dull, tired, and boring for NA), rated on a 4-point Likert scale (from "never felt" to "clearly felt") with score ranges of 5 to 20 . In this study, participants were asked to rate how much they experienced each emotion during the past week. The alphas were .89 and .77 for the PA and NA scales in this study, respectively. The concurrent validity of these subscales was 
established by Terasaki et al. (1992). Finally, the affect balance scores were calculated by subtracting the NA score from the PA score (PA-NA) or by dividing PA by NA (PA/NA).

Short-Term Life Satisfaction (LS). LS was assessed by means of the Short-term Life Satisfaction Scale (SLSS), which was translated into Japanese for this study. Although the SLSS includes similar instructions and contains five items similar to the Satisfaction with Life Scale (SWLS: Pavot \& Diener, 1993), these elements were revised to allow for asking about only the previous week instead of the whole past life. Participants answered the five items pertaining to life during the past week on a 7-point Likert scale ranging from 1 (strongly disagree) to 7 (strongly agree). Item examples included: "If I could relive the past week again, I would change almost nothing" and "I am satisfied with my life." Total scores ranged from 5 to 35 . The alpha was .87 in this study. The original scale (SLSS) has high reliability and both convergent and discriminant validity (Pavot \& Diener, 1993).

\section{Results}

Table 1 shows the correlations between all measures for both men and women, along with the means and standard deviations for each measure. Raw PA and NA scores were omitted from this table (for these data see Yamasaki, Sasaki, Uchida, \& Katsuma, 2011). Short-term LS showed significantly positive correlations with all of the affect balance measures. No significant differences in these correlations (PA-NA vs. LS and PA/NA vs. LS) between activated and deactivated affect were found, $t \mathrm{~s}(181)=.75$ and $1.59, p \mathrm{~s}>.10$, respectively, for men, and $t \mathrm{~s}(211)=1.09$ and $1.60, p \mathrm{~s}$ $>.10$, respectively, for women. With respect to the differences in the correlations between affect balance measures (PA-NA and PA/NA) and LS, two significant differences were found: for activated affect in men, the correlation between PA-NA and LS was significantly higher than between PA/NA and LS, $t(181)=2.47, p<.05$, while for

Table 1. Correlations between All Measures with Means and Standard Deviations (SD) for Men and Women

\begin{tabular}{|c|c|c|c|c|c|c|c|c|c|}
\hline \multirow[b]{2}{*}{ Variables } & \multirow[b]{2}{*}{1} & \multirow[b]{2}{*}{2} & \multirow[b]{2}{*}{3} & \multirow[b]{2}{*}{4} & \multirow[b]{2}{*}{5} & \multicolumn{2}{|c|}{ Men } & \multicolumn{2}{|c|}{ Women } \\
\hline & & & & & & Mean & $(S D)$ & Mean & $(S D)$ \\
\hline 1. Life Satisfaction & & $.43 * *$ & $.36^{* *}$ & $.48^{* *}$ & $.47 * *$ & 15.24 & $(5.86)$ & 15.28 & $(6.92)$ \\
\hline 2. Activated PA-NA & $.62 * *$ & & $.91 * *$ & $.44 * *$ & $.44 * *$ & .88 & $(9.37)$ & -.62 & (11.07) \\
\hline 3. Activated PA/NA & $.60^{* *}$ & $.92 * *$ & & $.38 * *$ & $.42 * *$ & 1.14 & $(.53)$ & 1.09 & (.51) \\
\hline 4. Deactivated PA-NA & $.57^{* *}$ & $.60 * *$ & $.57^{* *}$ & & $.94 * *$ & -2.15 & $(4.90)$ & -3.04 & (5.15) \\
\hline 5. Deactivated PA/NA & $.52 * *$ & $.55^{* *}$ & $.55^{* *}$ & $.94 * *$ & & .91 & $(.41)$ & .84 & (.40) \\
\hline
\end{tabular}

Note. $N=(184$ men and 214 women). Correlations shown above the diagonal are for men, and those shown below the diagonal are for women. $* p<.05 . * * p<.01$.

Table 2. Predicting Short-Term Life Satisfaction via Activated Positive and Negative Affect

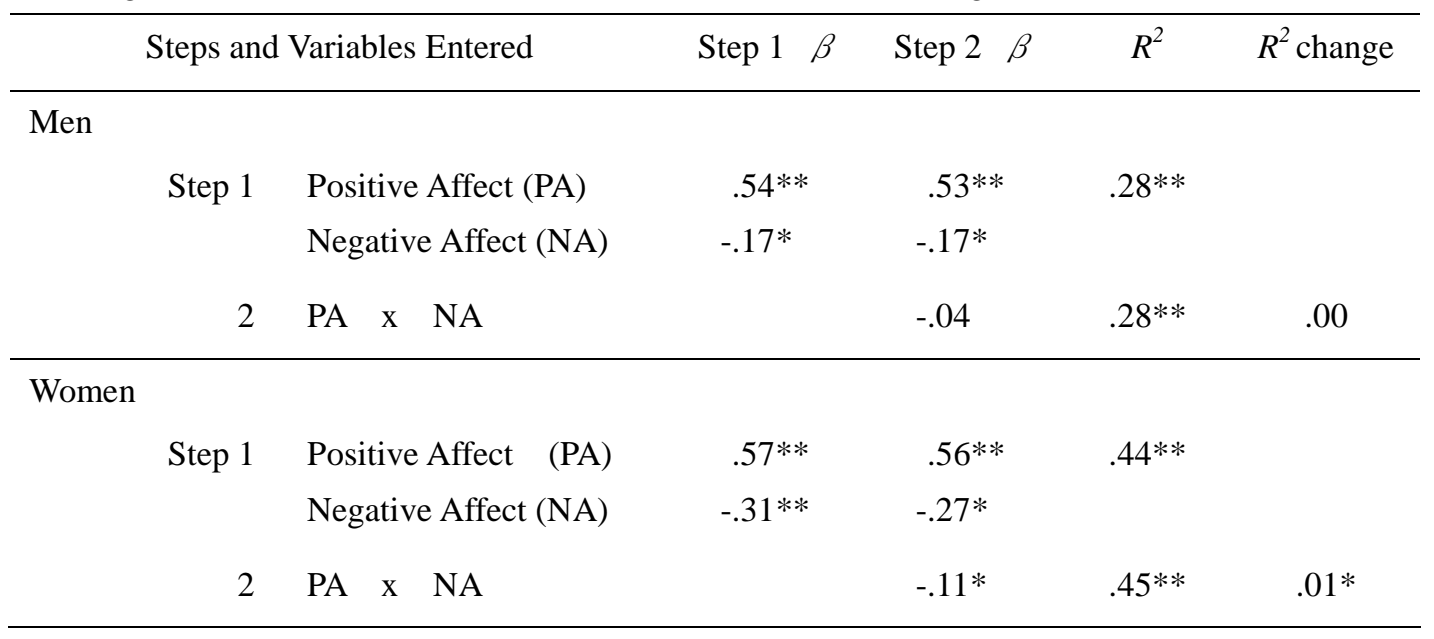

Note. $N=(184$ men and 214 women $) . * p<.05 . * * p<.01$. 
Table 3. Predicting Short-Term Life Satisfaction via Deactivated Positive and Negative Affect

\begin{tabular}{|c|c|c|c|c|c|}
\hline Steps an & Variables Entered & Step $1 \beta$ & Step $2 \beta$ & $R^{2}$ & $R^{2}$ change \\
\hline \multicolumn{6}{|l|}{ Men } \\
\hline Step 1 & Positive Affect (PA) & $.31^{* *}$ & $.30^{* *}$ & $.24 * *$ & \\
\hline & Negative Affect (NA) & $-.40 * *$ & $-.40 * *$ & & \\
\hline 2 & PA $x$ NA & & -.10 & $.25 * *$ & .01 \\
\hline \multicolumn{6}{|l|}{ Women } \\
\hline Step 1 & Positive Affect (PA) & $.32 * *$ & $.32 * *$ & $.35 * *$ & \\
\hline & Negative Affect (NA) & $-.51 * *$ & $-.51 * *$ & & \\
\hline 2 & PA $x$ NA & & .06 & $.36 * *$ & .00 \\
\hline
\end{tabular}

Note. $N=(184$ men and 214 women $) . \quad * p<.05 . * * p<.01$.

deactivated affect in women, the correlation between PA-NA and LS was significantly higher than between PA/NA and LS, $t(211)=2.56, p<.05$. No significant gender differences were found in any measures.

Scattergrams that compared individual values of affect balance (PA-NA) and short-term LS for activated and deactivated affect in men and women suggest positive linear relationships between affect balance and LS, i.e., higher affect balance corresponds to higher LS scores, for both affect in both men and women. Similar trends were also found using another measure of affect balance, PA/NA.

Next, in order to investigate the interactive effects of PA and NA on short-term LS, hierarchical regression analyses were conducted with short-term LS regressed on PA, NA, and PA x NA for activated affect (Table 2) and deactivated affect (Table 3). Following the recommendations of Aiken and West (1991), the predictor variables were standardized to avoid multicollinearity between the predictors and the interaction terms. In the regression analyses, PA and NA were entered on the first step, followed by ES on the second step, and then PA x ES and NA x ES on the third step.

Regarding activated affect (Table 2), a significant interaction between PA and NA was found along with a significant $R^{2}$ change in women. In comparison, no significant interaction between PA and NA was found in regards to deactivated affect (Table 3). PA had significantly positive associations with LS in terms of both activated and deactivated affect,

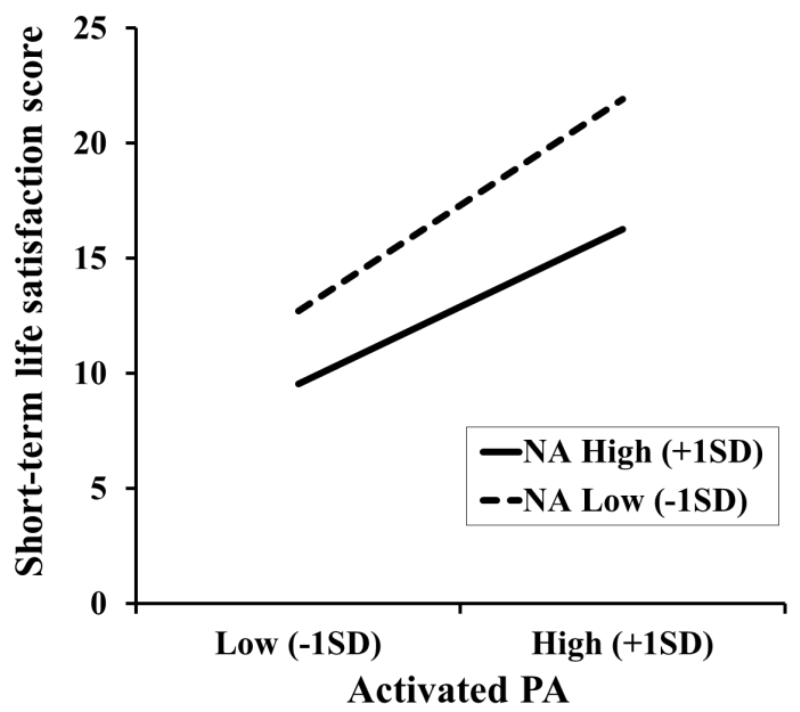

Figure 1. Scores on short-term life satisfaction as a function of activated negative affect (NA) and positive affect (PA) in women. 
while NA had significantly negative associations with LS. These main effects of PA and NA were detailed in Yamasaki et al. (2011).

The significant interaction of PA and NA was plotted using the procedure proposed by Aiken and West (1991) in Fig. 1. The predicted values on LS were computed on the basis of the scores for PA and NA of $1 S D$ below the mean and $1 S D$ above the mean. Both slopes were significant, $t \mathrm{~s}(210)=7.03$ for high NA and 10.51 for low NA, $p \mathrm{~s}<.01$. These results suggest that although activated PA had a significantly positive association with LS regardless of activated NA levels, this association was more positive when NA was lower.

\section{Discussion}

The current study focused on the relationship between affect balance and short-term LS taking into account the dimension of affect activation. Correlational analyses showed that short-term LS was positively associated with affect balance (PA-NA and PA/NA) in both men and women, regardless of activated and deactivated characteristics. Moreover, scattergrams plotting the relationship between affect balance and LS revealed a positive linear relationship, suggesting that higher affect balance linearly leads to higher short-term LS. Taken together, the current data suggest that regardless of levels of activation, higher PA/lower NA scores are associated with healthier and more adaptive statuses in non-clinical samples.

Moreover, the present finding that activated PA and NA had a significant interactive effect on short-term LS in women deserves note. The basis for the interaction was that although higher activated PA was associated with higher LS, this positive association was larger when NA was lower. This finding is inconsistent with some previous studies. For example, Cheng (2006) showed that the enhancing effect of PA on well-being appraisal is stronger when NA is higher, and Van Yeperen (2003) found similar effects on job performance. Comparatively, Yamasaki, Nagai, and Uchida (2007) showed that health status as measured using the General Health Questionnaire (Goldberg \& Hiller, 1979) was improved more by PA when NA was lower, which is consistent with the present data. Since significant interactions between PA and NA were found in activated affect in all of the above studies as well as in the current study, the causes of these other discrepancies remain unclear. However, since the detailed methods (including specific measures used and participant types) are different among the studies, future research should carefully examine how methodological conditions influence results. Additionally, clarification of the reasons why our finding did not pertain to men or with regard to deactivated affect would also be a future research avenue worth pursuing.

Some limitations of the present research should be noted. First, the cross-sectional design used in this study should be replaced by longitudinal or prospective designs so that causal relations might be tested. Particularly in prospective designs, short-term LS might be a more effective endpoint compared to global extant LS since the former is more sensitive to current conditions. Second, the concept and classification of LS should be further scrutinized. Although LS represents a hot topic in recent research, it seems likely that this concept has ambiguous and multiple components. For instance, Kim and colleagues (Kim, 2004; Jang \& Kim, 2009) introduced the concept of "implicit LS" in contrast to the predominant concept of LS that they termed "explicit LS". Implicit LS is measured by assessing the strength of automatic associations of my life with Good- versus Bad-related words (Kim, 2004). According to their theory, explicit LS reflects current situations and may not necessarily reveal individuals' summed experiences throughout their life. In contrast, implicit LS is less influenced by current situations and may better reflect experiences in the past whole life. In this study, a state-like, short-term LS was measured. As such, LS would be influenced more by current situations, which was our goal in the present study. LS could have both trait-like and state-like characteristics, as many other psychological concepts do. Short-term LS is regarded as a measure that reflects the state component of LS. However, since no prior research has compared global (explicit) and short-term LS, the comparative examination of both types of LS represents an urgent research topic.

Third, affect should be ecologically measured in longitudinal or prospective studies, e.g., by using experience-time sampling methods. Since affect is easily changed, repeated measurements would be needed to more accurately assess experiences of PA and NA. Finally, by directly evaluating and comparing these health measures in clinical samples or in individuals at high risk for health impairment, we should conduct longitudinal research to conclude whether similar findings are consistently observed. If we find similar roles of activated/deactivated affect in future research, we could then move forward towards prevention or interventional research that might enhance or improve health and adjustment in practical settings.

\section{Acknowledgements}

The authors are grateful to Dr. Megumi Sasaki for assistance in conducting this research. This research was supported by a grant from the Japan Society of the Promotion of Science (No. 21530730). 


\section{References}

Aiken, L. S., \& West, S. G. (1991). Multiple Regression: Testing and interactions. Newbury Park, CA: Sage.

Cheng, S. T. (2006). Negative emotions make positive emotions more salient in well-being appraisal. Personality and Individual Differences, 40, 557-567. http://dx.doi.org/10.1016/j.paid.2005.08.002

De Dreu, C.K.W., Baas, M., \& Nijstad, B. A. (2008). Hedonic tone and activation level in the mood-creativity link toward a dual pathway to creativity model. Journal of Personality and Social Psychology, 94, 739-756. http://dx.doi.org/10.1037/0022-3514.94.5.739

Goldberg, D. P., \& Hillier, V. F. (1979). A scaled version of the General Health Questionnaire. Psychological Medicine, 9, 139-145. http://dx.doi.org/10.1017/S0033291700021644

Han, J. Y., Shaw, B. R., Hawkins, R. P., Pingree, S., McTavish, F., \& Gustafson, D. H. (2008). Expressing positive emotions within online support groups by women with breast cancer. Journal of Health Psychology, 13, 1002-1007. http://dx.doi.org/10.1177/1359105308097963

Jang, D., \& Kim, D. Y. (2009). Two faces of human happiness: Explicit and implicit life satisfaction. Asian Journal of Social Psychology, 12, 185-198. http://dx.doi.org/10.1111/j.1467-839X.2009.01284.x

Keyes, C. L. M. (2002). The mental health continuum: From languishing to flourishing in life. Journal of Health and Social Behavior, 43, 207-222. http://dx.doi.org/10.2307/3090197

Kim, D-Y. (2004). The implicit life satisfaction measure. Asian Journal of Social Psychology, 7, $236-262$. http://dx.doi.org/10.1111/j.1467-839X.2004.00148.x

Koydemir, S., Şimşek, Ö. F., Schütz, A., \& Tipandjan, A. (2013). Differences in how trait emotional intelligence predicts life satisfaction: The role of affect balance versus social support in India and Germany. Journal of Happiness Studies, 14, 51-66. http://dx.doi.org/10.1007/s10902-011-9315-1

Larsen, R. J., \& Diener, E. (1992). Promises and problems with the circumplex model of emotion. In M.S. Clard (Ed.), Review of personality and social Psychology: Emotion. Newbury Park, CA: Sage, 13, 25-59.

Nezlek, J. B., \& Kuppens, P. (2008). Regulating positive and negative emotions in daily life. Journal of Personality, 76, 561-579. http://dx.doi.org/10.1111/j.1467-6494.2008.00496.x

Pavot, W., \& Diener, E. (1993). Review of the satisfaction with life scale. Psychological Assessment, 5, $164-172$. http://dx.doi.org/10.1037/1040-3590.5.2.164

Pettit, J.W., Kline, J. P., Gencoz, T., Gencoz, F., \& Joiner, T. E. (2001). Are happy people healthier? The specific role of positive affect in predicting self-reported health symptoms. Journal of Research in Personality, 35, 521-536. http://dx.doi.org/10.1006/jrpe.2001.2327

Pressman, S. D., \& Cohen, S. (2005). Does positive affect influence health? Psychological Bulletin, 131, $925-971$. http://dx.doi.org/10.1037/0033-2909.131.6.925

Russell, J. A. (1980). A circumplex model of affect. Journal of Personality and Social Psychology, 39, 1161-1178. http://dx.doi.org/10.1037/h0077714

Sanjuán, P. (2011). Affect balance as mediating variable between effective psychological functioning and satisfaction with life. Journal of Happiness Studies, 12, 373-384. http://dx.doi.org/10.1007/s10902-010- 9199-5

Sato, A., \& Yasuda, A. (2001). Development of the Japanese version of Positive and Negative Affect Schedule (PANAS) scales. Japanese Journal of Personality, 9, 138-189.

Schimmack, U., Radhakrishnan, P., Oishi, S., Dzokoto, V., \& Ahadi, S. (2002). Culture, personality, and subjective well-being: Integrating process models of life satisfaction. Journal of Personality and Social Psychology, 82, 582-593. http://dx.doi.org/10.1037/0022-3514.82.4.582

Suh, E., Diener, E., Oishi, S., \& Triandis, H.C. (1998). The shifting basis of life satisfaction judgments across cultures: Emotions versus norms. Journal of Personality and Social Psychology, 74, 482-493. http://dx.doi.org/10.1037/0022-3514.74.2.482

Terasaki, S., Kishimoto, Y., \& Koga, A. (1992). Construction of a multiple mood scale. Japanese Journal of Psychology, 62, 350-356. http://dx.doi.org/10.4992/jjpsy.62.350

Tsai, J.L., Knutson, B., \& Fung, H.H. (2006). Cultural variation in affect valuation. Journal of Personality and Social Psychology, 90, 288-307. http://dx.doi.org/10.1037/0022-3514.90.2.288

Van-Yperen, N. W. (2003). On the link between different combinations of negative affectivity (NA) and positive 
affectivity (PA) and job performance. Personality and Individual Differences, 35, 1873-181. http://dx.doi.org/10.1016/S0191-8869(03)00036-9

Watson, D., Clark, L. A., \& Tellegen, A. (1988). Development and validation of brief measures of Positive and Negative Affect: The PANAS Scales. Journal of Personality and Social Psychology, 54, 1063-1070. http://dx.doi.org/10.1037/0022-3514.54.6.1063

Yamasaki, K., Nagai, A., \& Uchida, K. (2007). A longitudinal study of the relationship between affect and both health and lifestyle. Psychologia, 50, 177-191. http://dx.doi.org/10.2117/psysoc.2007.177

Yamasaki, K., Sasaki, M., Uchida, K., \& Katsuma, L. (2011). Effects of positive and negative affect and emotional suppression on short-term life satisfaction. Psychology, Health \& Medicine, 16, 313-322. http://dx.doi.org/10.1080/13548506.2011.554564

Zhu, H. (2015). Social support and affect balance mediate the association between forgiveness and life satisfaction. Social Indicators Research, 124, 671-681. http://dx.doi.org/10.1007/s11205-014-0790-8

\section{$(\mathrm{cc}) \mathrm{BY}$}

This work is licensed under a Creative Commons Attribution 3.0 License. 